\title{
Estudos de SAR e Docking Molecular para o desenvolvimento de novos inibidores da
}

\section{Tirosinase}

\author{
SAR and Molecular Docking Studies for the development of new Tyrosinase inhibitors \\ Estudios de SAR y de Acoplamiento Molecular para el desarrollo de nuevos inhibidores de
}

Tirosinasa

Recebido: 08/01/2022 | Revisado: 12/01/2022 | Aceito: 20/01/2022 | Publicado: 06/02/2022

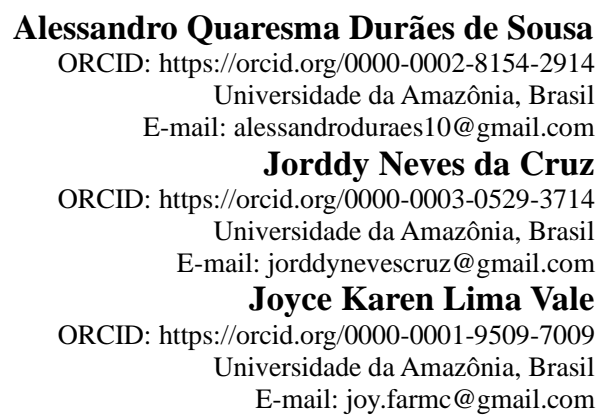

\begin{abstract}
Resumo
A tirosinase é uma enzima que participa da biossíntese da melanina. Logo, é considerada uma enzima muito importante para alguns processos fisiológicos, além disso, está envolvida desde o processo de escurecimento de algumas frutas até a neurodegeneração do Parkinson. Assim, o ácido kójico é um importante metabólito que possui a propriedade de inibição da enzima tirosinase em relação a produção de melanina. Com isso, o ácido kójico é muito utilizado na medicina, caracterizando-se dessa forma como importante para certos tipos de tratamentos. Sendo assim, neste projeto utilizamos abordagens de modelagem molecular para análises de derivados do ácido kójico, alvo molecular contra a enzima tirosinase, inicialmente a otimização e parametrização foram realizadas através do método semi-empírico PM3. Posteriormente os métodos foram otimizados pelo método TFD, em seguida utilizando o programa computacional MVD foi realizado os cálculos de energia para as moléculas. Tendo como guia para a definição de parâmetros estruturais a estrutura do Tropolone para as análises computacionais visando o ácido kójico e seus derivados. Dessa forma, os resultados obtidos nas simulações chegaram à satisfação. Baseado nos valores de energia obtidos através das análises, os derivados 2,3,8 e 9 são sugeridos como moléculas potenciais candidatos a inibidores da tirosina.
\end{abstract}

Palavras-chave: Tirosina; Ácido kojico; Novos inibidores; Modelagem molecular; SAR; Docking.

\begin{abstract}
Tyrosinase is an enzyme that participates in melanin biosynthesis. Therefore, it is considered a very important enzyme for some physiological processes, in addition, it is involved from the browning process of some fruits to Parkinson's neurodegeneration. Thus, kojic acid is an important metabolite that has the property of inhibiting the tyrosinase enzyme in relation to the production of melanin. Thus, kojic acid is widely used in medicine, thus characterizing itself as important for certain types of treatments. Therefore, in this project we use molecular modeling approaches to analyze kojic acid derivatives, a molecular target against the tyrosinase enzyme, initially the optimization and parameterization were performed using the semi-empirical PM3 method. Subsequently, the methods were optimized by the TFD method, then using the MVD computer program, the energy calculations for the molecules were performed. Taking as a guide for the definition of structural parameters the Tropolone structure for computational analyzes targeting kojic acid and its derivatives. Thus, the results obtained in the simulations reached satisfaction. Based on the energy values obtained through the analysis, derivatives 2,3,8 and 9 are suggested as potential candidate molecules for tyrosine inhibitors.
\end{abstract}

Keywords: Tyrosine; Kojic acid; New inhibitors; Molecular modeling; SAR; Docking.

\section{Resumen}

La tirosinasa es una enzima que participa en la biosíntesis de melanina. Por ello, se considera una enzima muy importante para algunos procesos físiológicos, además, interviene desde el proceso de pardeamiento de algunas frutas hasta la neurodegeneración del Parkinson. Así, el ácido kójico es un metabolito importante que tiene la propiedad de 
inhibir la enzima tirosinasa en relación con la producción de melanina. Así, el ácido kójico es ampliamente utilizado en medicina, caracterizándose así como importante para cierto tipo de tratamientos. Por lo tanto, en este proyecto utilizamos enfoques de modelado molecular para el análisis de derivados del ácido kójico, un objetivo molecular contra la enzima tirosinasa, inicialmente la optimización y parametrización se realizaron utilizando el método semiempírico PM3. Posteriormente, los métodos fueron optimizados por el método TFD, luego, utilizando el programa informático MVD, se realizaron los cálculos de energía para las moléculas. Teniendo como guía para la definición de parámetros estructurales la estructura de la Tropolona para los análisis computacionales con vistas al ácido kójico y sus derivados. Así, los resultados obtenidos en las simulaciones alcanzaron la satisfacción. Con base en los valores de energía obtenidos a través de los análisis, los derivados 2,3,8 y 9 se sugieren como moléculas candidatas potenciales para inhibidores de tirosina.

Palabras clave: Tirosina; Ácido kójico; Nuevos inhibidores; Modelado molecular; SAR; Acoplamiento.

\section{Introdução}

A tirosinase (EC 1.14.18.1) é uma enzima binuclear que contém um cobre como cofator redox no seu sítio ativo, e através de reações de hidroxilação, (atividade monofenolase), oxidação (atividade difenolase), seguida de polimerização, forma melanina (Li et al., 2019; Abbas et al., 2017). A tirosinase, está envolvida tanto nos processos de escurecimento de frutos, quanto em mecanismos de neurodegeneração de Parkinson (Yimei Xu et al., 1997). A produção de melanina pela tirosinase é essencial para a proteção da pele frente às radiações solares (Maeda \& Fukuda, 1991).

A melanina consiste em uma substância química que se apresenta na forma de compostos poliméricos que são derivados da tirosina presente na maioria dos reinos de seres vivos (Cordero \& Casadevall, 2020), constituindo-se em um pigmento escuro produzido em organelas elípticas, que por sua vez são especializadas na síntese de melanina, conhecidas como melanossomas localizadas dentro de células situadas principalmente na epiderme, conhecidas como melanócitos. Tal pigmentação é ocasionada pela enzima tirosinase na execução da melanogênese (Cichorek et al., 2013).

A melanina não consiste em uma substância única e isolada, mas a mesma compõe-se em um grupo de várias substâncias, possuindo entre elas propriedades semelhantes. Dessa forma, são estruturas escurecidas ocasionadas pela polimerização oxidativa de indólicos e fenólicos, estado agregadas a proteínas e carboidratos podem ser encontradas em animais, plantas e microrganismos (de Paulo Farias et al., 2020; Krepsky et al., 2009).

A enzima tirosinase responsável em desempenhar a biossíntese da melanina se compreende em três domínios: o domínio interno, sendo responsável pelo desempenho catalítico, contém resíduos de histidina, onde tais resíduos realizam ligações a íons de cobre, atuando no transporte da enzima (H. Lee et al., 2014; S.-R. Lee et al., 2005).

O Ácido kójico (AKJ) consiste em um metabólito natural produzidos por alguns fungos tendo a capacidade de inibir a ação da enzima tirosinase na produção de melanina. Assim, o AKJ e outros derivados são utilizados em diversos objetivos na medicina dentre elas a radioproteção contra raios solares e ação clareadora de pele em forma de creme são algumas delas(Saeedi et al., 2019).

Diante disso, o AKJ e seus derivados são amplamente utilizados na medicina para diversas finalidade e tratamento, evidenciando a sua importância na buscar e elucidação do funcionamento de tais molécula frente a processos ainda não totalmente elucidados. O Ácido Kójico possui amplo perfil farmacológico, é tido padrão positivo para tirosinase(Saghaie et al., 2013).

O Ácido kójico inibe a etapa monofelase de forma competitiva e a etapa difenolase por inibição mista Dessa forma, o AKJ possui a capacidade de desempenhar uma afinidade com o sítio ativo da enzima tirosinase, ocorrendo quelação com íons CU+2 que estão presentes. Além disso, em um sítio ativo diferente ocorre uma ligação, onde é realizado uma inibição nãocompetitiva (Schurink et al., 2007; Sendovski et al., 2011).

No entanto, o AKJ é utilizado de forma limitada pela indústria de cosméticos, devido sua toxicidade aumentando a possibilidade de possíveis reações adversas, ou seja, reações não desejadas, tornando algo perigoso à saúde do paciente a qual 
utiliza, e pela instabilidade das formulações produzidas, assim é necessário uma limitação da utilização dessa substância (Saghaie et al., 2013).

Estes estudos ressaltam a importância do desenvolvimento de novos inibidores de tirosinase derivados do AKJ, pois, inúmeros compostos têm sido relatados na atualidades, mas, a maioria não são potentes o suficiente para proporcionar o inibição desejada, ou não possuem perfis farmacológico terapêuticos adequados para determinados tratamentos (Wen et al., 2010; You Xu et al., 2011; You et al., 2015).

A modelagem molecular permiti a criação, manipulação de modelos representativos fidedignos as estruturas reais, permitindo também a realização de cálculos físico-químicos (Araújo et al., 2020; da Silva Júnior et al., 2021; Leão et al., 2020; Neto et al., 2020; Santana de Oliveira et al., 2020). Logo, a modelagem molecular é uma ferramenta para produção direta e indireta de potencias candidatos a fármacos. Sendo de forma indireta quando não é de conhecimento a estrutura representativo receptor, que possibilitariam parâmetros para a elucidação da relação estrutura atividade do alvo trabalhado em questão (Almeida et al., 2021; Castro et al., 2021; Lima et al., 2020; Thomsen \& Christensen, 2006). E de forma direta quando se é conhecida a forma tridimensional do alvo biológico em questão trabalhado, possibilitando assim compreender as interações envolvidas no complexo em questão.

\section{Metodologia}

\subsection{Teoria do funcional de densidade}

A otimização da geometria e obtenção de parâmetros teóricos foi realizada através do método semi-empírico PM3 (Terceira Parametrização). As geometrias obtidas pelo método PM3 foram otimizadas pelo método da Teoria do Funcional de Densidade (TFD) usando o conjunto de base 6-31G (d,p). Somente as conformações mais estáveis para as moléculas estudadas forma utilizadas. Nesta etapa, os cálculos químicos para os derivados foram realizados utilizando os Gaussian09 (Frisch et al., 2009).

\subsection{Molecular docking}

Através do programa Molegro Virtual Docker (MVD®) (Neves Cruz et al., 2020; Ramos et al., 2020; Santos et al., 2020), foram realizados cálculos de energia de interação, do AKJ e 12 derivados, no sítio ativo da enzima tirosinase, utilizando o algoritmo MolDock $($ EScore $=$ Einter + Eintra), onde Einter é a energia de interação ligante-proteína e Eintra é a energia interna do ligante (Costa et al., 2020; Santana de Oliveira et al., 2021). A análise foi baseada no modelo cristalográfico da tirosinase tipo Agaricus bisporus (Data Bank Protein, código PDB: 2Y9X). A estrutura do Tropolone (OTR) foi submetida ao redocking e utilizada como guia para definir os parâmetros estruturais e validar a estratégia computacional no docking do AKJ e derivados.

\section{Resultados e Discussão}

Os derivados planejados são exibidos na Figura 1. 
Figura 1. Derivados no ácido kojico. Os átomos representados em cinza são átomos de carbono, em vermelho átomos de oxigênio, em azul escuro átomos de nitrogênio, em azul claro átomos de hidrogênio e em verde átomos de cloro.
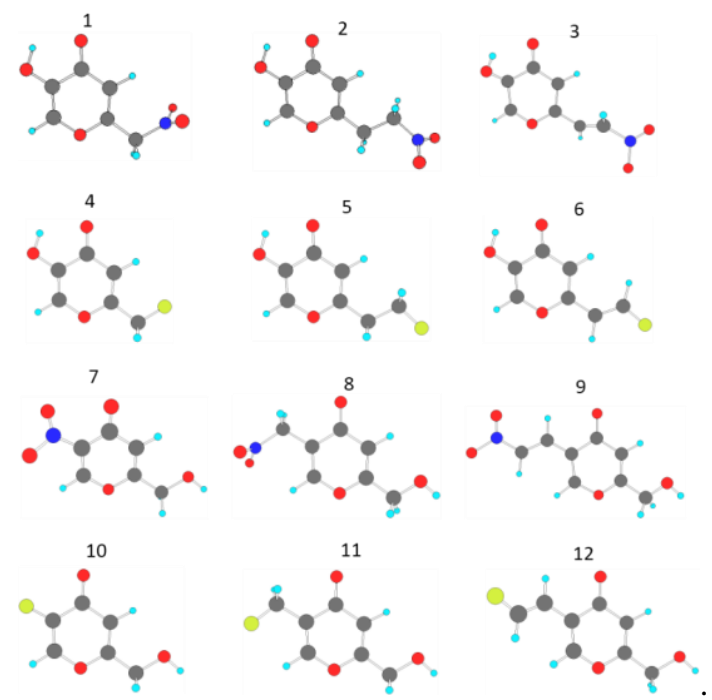

Fonte: Autores.

Durante as simulações observou-se que as orientações vinculativas e geometrias para o OTR acoplado no sítio ativo 2Y9X pode ser sobreposta e são consistentes com a estrutura de raios-X publicada exibida na Figura 2.

Figura 2. Docking do AKJ no sítio ativo da tirosinase.

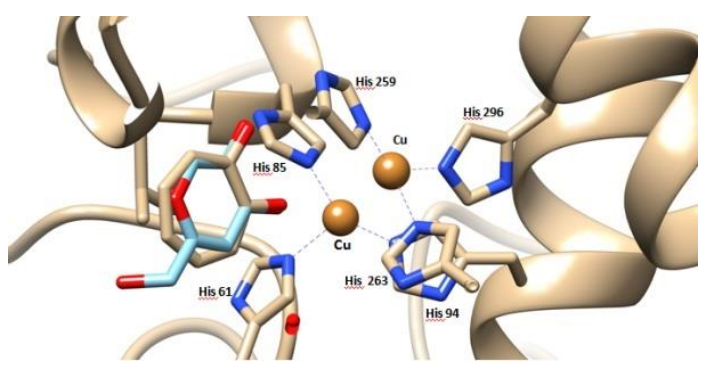

Fonte: Autores.

O AKJ e os 12 derivados testados foram ancorados na mesma região que o inibidor OTR. Esses resultados são consistentes com modelos obtidos para ancoragem em tirosinase de A. bisporus2. Além disso, demonstraram boa afinidade com o sítio receptor e menor energia quando comparados ao inibidor (OTR: -57.478kcal.mol-1). Os derivados 2,3,8 e 9 demonstraram valores de MolDock Score (kcal.mol-1) de -71.3983, -70.0027, -71.6849 e -72.6554, respectivamente. Estes resultados são corroborados por estudos anteriores que sugerem que a introdução de elementos eletronegativos pode contribuir para a ligação do possível inibidor à cadeia lateral da enzima.

\section{Conclusão}

Os resultados das simulações de Docking foram satisfatórios. Os valores de energia obtidos sugerem que os derivados 2,3,8 e 9 podem ser potenciais candidatos a novos inibidores da tirosinase. 


\section{Referências}

Abbas, F., Ke, Y., Yu, R., Yue, Y., Amanullah, S., Jahangir, M. M., \& Fan, Y. (2017). Volatile terpenoids: multiple functions, biosynthesis, modulation and manipulation by genetic engineering. Planta, 246(5), 803-816. https://doi.org/10.1007/s00425017-2749-x

Almeida, V. M., Dias, Ê. R., Souza, B. C., Cruz, J. N., Santos, C. B. R., Leite, F. H. A., Queiroz, R. F., \& Branco, A. (2021). Methoxylated flavonols from Vellozia dasypus Seub ethyl acetate active myeloperoxidase extract: in vitro and in silico assays. Journal of Biomolecular Structure and Dynamics, 1-10. https://doi.org/10.1080/07391102.2021.1900916

Araújo, P. H. F., Ramos, R. S., da Cruz, J. N., Silva, S. G., Ferreira, E. F. B., de Lima, L. R., Macêdo, W. J. C., Espejo-Román, J. M., Campos, J. M., \& Santos, C. B. R. (2020). Identification of Potential COX-2 Inhibitors for the Treatment of Inflammatory Diseases Using Molecular Modeling Approaches. Molecules, $25(18)$, 4183. https://doi.org/10.3390/molecules25184183

Castro, A. L. G., Cruz, J. N., Sodré, D. F., Correa-Barbosa, J., Azonsivo, R., de Oliveira, M. S., de Sousa Siqueira, J. E., da Rocha Galucio, N. C., de Oliveira Bahia, M., Burbano, R. M. R., do Rosário Marinho, A. M., Percário, S., Dolabela, M. F., \& Vale, V. V. (2021). Evaluation of the genotoxicity and mutagenicity of isoeleutherin and eleutherin isolated from Eleutherine plicata herb. using bioassays and in silico approaches. Arabian Journal of Chemistry, 14(4), 103084. https://doi.org/10.1016/j.arabjc.2021.103084

Cichorek, M., Wachulska, M., Stasiewicz, A., \& Tymińska, A. (2013). Skin melanocytes: biology and development. Advances in Dermatology and Allergology, 1, 30-41. https://doi.org/10.5114/pdia.2013.33376

Cordero, R. J. B., \& Casadevall, A. (2020). Melanin. Current Biology, 30(4), R142-R143. https://doi.org/10.1016/j.cub.2019.12.042

Costa, E. B., Silva, R. C., Espejo-Román, J. M., Neto, M. F. de A., Cruz, J. N., Leite, F. H. A., Silva, C. H. T. P., Pinheiro, J. C., Macêdo, W. J. C., \& Santos, C. B. R. (2020). Chemometric methods in antimalarial drug design from 1,2,4,5-tetraoxanes analogues. SAR and QSAR in Environmental Research, 31(9), 677-695. https://doi.org/10.1080/1062936X.2020.1803961

da Silva Júnior, O. S., Franco, C. de J. P., de Moraes, A. A. B., Cruz, J. N., da Costa, K. S., do Nascimento, L. D., \& Andrade, E. H. de A. (2021). In silico analyses of toxicity of the major constituents of essential oils from two Ipomoea L. species. Toxicon, 195, 111-118. https://doi.org/10.1016/j.toxicon.2021.02.015

de Paulo Farias, D., Neri-Numa, I. A., de Araújo, F. F., \& Pastore, G. M. (2020). A critical review of some fruit trees from the Myrtaceae family as promising sources for food applications with functional claims. Food Chemistry, $306,125630$. https://doi.org/10.1016/j.foodchem.2019.125630

Krepsky, P. B., Cervelin, M. de O., Porath, D., Peters, R. R., Ribeiro-do-Valle, R. M., \& Farias, M. R. (2009). High performance liquid chromatography determination of cucurbitacins in the roots of Wilbrandia ebracteata Cogn. Revista Brasileira de Farmacognosia, 19(3). https://doi.org/10.1590/S0102-695X2009000500011

Leão, R. P., Cruz, J. V., da Costa, G. V., Cruz, J. N., Ferreira, E. F. B., Silva, R. C., de Lima, L. R., Borges, R. S., dos Santos, G. B., \& Santos, C. B. R. (2020). Identification of New Rofecoxib-Based Cyclooxygenase-2 Inhibitors: A Bioinformatics Approach. Pharmaceuticals, 13(9), 209. https://doi.org/10.3390/ph13090209

Lee, H., Kim, Y., Park, A., \& Nam, J.-M. (2014). Amyloid- $\beta$ Aggregation with Gold Nanoparticles on Brain Lipid Bilayer. Small, 10(9), 1779-1789. https://doi.org/10.1002/smll.201303242

Lee, S.-R., Park, J.-H., Park, E. K., Chung, C. H., Kang, S.-S., \& Bang, O.-S. (2005). Akt-induced promotion of cell-cycle progression at G2/M phase involves upregulation of NF-Y binding activity in PC12 cells. Journal of Cellular Physiology, 205(2), 270-277. https://doi.org/10.1002/jcp.20395

Li, Z., Chen, Y., Yang, Y., Yu, Y., Zhang, Y., Zhu, D., Yu, X., Ouyang, X., Xie, Z., Zhao, Y., \& Li, L. (2019). Recent Advances in Nanomaterials-Based Chemo-Photothermal Combination Therapy for Improving Cancer Treatment. Frontiers in Bioengineering and Biotechnology, 7. https://doi.org/10.3389/fbioe.2019.00293 
Lima, A. de M., Siqueira, A. S., Möller, M. L. S., Souza, R. C. de, Cruz, J. N., Lima, A. R. J., Silva, R. C. da, Aguiar, D. C. F., Junior, J. L. da S. G. V., \& Gonçalves, E. C. (2020). In silico improvement of the cyanobacterial lectin microvirin and mannose interaction. Journal of Biomolecular Structure and Dynamics, 1-10. https://doi.org/10.1080/07391102.2020.1821782

Maeda, K., \& Fukuda, M. (1991). In vitro effectiveness of several whitening cosmetic components in human melanocytes. Journal of the Society of Cosmetic Chemists, 42(6), 361-368.

M.J. Frisch, G.W. Trucks, H.B. Schlegel, G.E. Scuseria, M.A. Robb, J.R. Cheeseman, V. Barone, B. Mennucci, G.A. Petersson, H. Nakatsuji, M. Caricato, X.Li, H.P. Hratchian, A.F. Izmaylov, G. Zheng, J.L. Sonnenberg, M. Hada, M. Ehara, K. Toyota, R. Fukuda, J. Hasegawa, M. Ishida, T. Nakajima, O. Kitao, H.Nakai, T. Vreven, J.A. Montgomery, J.E. Peralta, F. Ogliaro, M. Bearpark, J.J. Heyd, E. Brothers, N. Kudin, V.N. Staroverov, R. Kobayashi, J. Normand, K.Raghavachari, A. Rendell, J.C. Burant, S.S. Iyengar, J. Tomasi, M. Cossi, N. Rega, J.M. Millam, M. Klene, J.E. Knox, J.B. Cross, V. Bakken, C. Adamo, J.Jaramillo, R. Gomperts, O. Yazyev, A.J. Austin, R. Cammi, C. Pomelli, J.W. Ochterski, R.L. Martin, K. Morokuma, V.G. Zakrzewski, A. Voth, P. Salvador,J.J. Dannenberg, S. Dapprich, A.D. Daniels, J.B. Foresman, J. v Ortiz, J. Cioslowski, D.J. Fox, D.J. Gaussian, J. Bloino, Y. Honda, K.N. Kudin, R.E.Stratmann, G.A. Voth, Gaussian 09, (2009) 2-3.

Neto, R. de A. M., Santos, C. B. R., Henriques, S. V. C., Machado, L. de O., Cruz, J. N., da Silva, C. H. T. de P., Federico, L. B., Oliveira, E. H. C. de, de Souza, M. P. C., da Silva, P. N. B., Taft, C. A., Ferreira, I. M., \& Gomes, M. R. F. (2020). Novel chalcones derivatives with potential antineoplastic activity investigated by docking and molecular dynamics simulations. Journal of Biomolecular Structure and Dynamics, 1-13. https://doi.org/10.1080/07391102.2020.1839562

Neves Cruz, J., da Costa, K. S., de Carvalho, T. A. A., \& de Alencar, N. A. N. (2020). Measuring the structural impact of mutations on cytochrome P450 21A2, the major steroid 21-hydroxylase related to congenital adrenal hyperplasia. Journal of Biomolecular Structure and Dynamics, 38(5), 1425-1434. https://doi.org/10.1080/07391102.2019.1607560

Ramos, R. S., Macêdo, W. J. C., Costa, J. S., da Silva, C. H. T. de P., Rosa, J. M. C., da Cruz, J. N., de Oliveira, M. S., de Aguiar Andrade, E. H., e Silva, R. B. L., Souto, R. N. P., \& Santos, C. B. R. (2020). Potential inhibitors of the enzyme acetylcholinesterase and juvenile hormone with insecticidal activity: study of the binding mode via docking and molecular dynamics simulations. Journal of Biomolecular Structure and Dynamics, 38(16), 4687-4709. https://doi.org/10.1080/07391102.2019.1688192

Saeedi, M., Eslamifar, M., \& Khezri, K. (2019). Kojic acid applications in cosmetic and pharmaceutical preparations. Biomedicine \& Pharmacotherapy, 110, 582-593. https://doi.org/10.1016/j.biopha.2018.12.006

Saghaie, L., Pourfarzam, M., Fassihi, A., \& Sartippour, B. (2013). Synthesis and tyrosinase inhibitory properties of some novel derivatives of kojic acid. Research in Pharmaceutical Sciences, 8(4), 233-242.

Santana de Oliveira, M., da Cruz, J. N., Almeida da Costa, W., Silva, S. G., Brito, M. da P., de Menezes, S. A. F., de Jesus Chaves Neto, A. M., de Aguiar Andrade, E. H., \& de Carvalho Junior, R. N. (2020). Chemical Composition, Antimicrobial Properties of Siparuna guianensis Essential Oil and a Molecular Docking and Dynamics Molecular Study of its Major Chemical Constituent. Molecules, 25(17), 3852. https://doi.org/10.3390/molecules25173852

Santana de Oliveira, M., Pereira da Silva, V. M., Cantão Freitas, L., Gomes Silva, S., Nevez Cruz, J., \& Aguiar Andrade, E. H. (2021). Extraction Yield, Chemical Composition, Preliminary Toxicity of Bignonia nocturna (Bignoniaceae) Essential Oil and in Silico Evaluation of the Interaction. Chemistry \& Biodiversity, 18(4). https://doi.org/10.1002/cbdv.202000982

Santos, C. B. R., Santos, K. L. B., Cruz, J. N., Leite, F. H. A., Borges, R. S., Taft, C. A., Campos, J. M., \& Silva, C. H. T. P. (2020). Molecular modeling approaches of selective adenosine receptor type 2A agonists as potential anti-inflammatory drugs. Journal of Biomolecular Structure and Dynamics, 1-13. https://doi.org/10.1080/07391102.2020.1761878

Schurink, M., van Berkel, W. J. H., Wichers, H. J., \& Boeriu, C. G. (2007). Novel peptides with tyrosinase inhibitory activity. Peptides, 28(3), 485-495. https://doi.org/10.1016/j.peptides.2006.11.023

Sendovski, M., Kanteev, M., Ben-Yosef, V. S., Adir, N., \& Fishman, A. (2011). First Structures of an Active Bacterial Tyrosinase Reveal Copper Plasticity. Journal of Molecular Biology, 405(1), $227-237$. https://doi.org/10.1016/j.jmb.2010.10.048 
Research, Society and Development, v. 11, n. 2, e19811225515, 2022

(CC BY 4.0) | ISSN 2525-3409 | DOI: http://dx.doi.org/10.33448/rsd-v11i2.25515

Thomsen, R., \& Christensen, M. H. (2006). MolDock: A New Technique for High-Accuracy Molecular Docking. Journal of Medicinal Chemistry, 49(11), 3315-3321. https://doi.org/10.1021/jm051197e

Wen, Z., Liu, B., Zheng, Z., You, X., Pu, Y., \& Li, Q. (2010). Preparation of liposomes entrapping essential oil from Atractylodes macrocephala Koidz by modified RESS technique. Chemical Engineering Research and Design, 88(8), 11021107. https://doi.org/10.1016/j.cherd.2010.01.020

Xu, Yimei, Stokes, A. H., Freeman, W. M., Kumer, S. C., Vogt, B. A., \& Vrana, K. E. (1997). Tyrosine mRNA is expressed in human substantia nigra. Molecular Brain Research, 45(1), 159-162. https://doi.org/10.1016/S0169-328X(96)00308-7

Xu, You, Shen, Z., Shen, J., Liu, G., Li, W., \& Tang, Y. (2011). Computational insights into the different catalytic activities of CYP2A13 and CYP2A6 on NNK. Journal of Molecular Graphics and Modelling, 30, 1-9. https://doi.org/10.1016/j.jmgm.2011.05.002

You, A., Zhou, J., Song, S., Zhu, G., Song, H., \& Yi, W. (2015). Structure-based modification of 3-/4-aminoacetophenones giving a profound change of activity on tyrosinase: From potent activators to highly efficient inhibitors. European Journal of Medicinal Chemistry, 93, 255-262. https://doi.org/10.1016/j.ejmech.2015.02.013 